\title{
Blueberry-enriched diet ameliorates age-related declines in NMDA receptor-dependent LTP
}

\author{
Steven J. Coultrap • Paula C. Bickford • \\ Michael D. Browning
}

Received: 29 January 2008 / Accepted: 26 June 2008 / Published online: 10 August 2008

(C) The Author(s) 2008

\begin{abstract}
NMDA receptor-dependent long-term potentiation (LTP) in the hippocampus is widely accepted as a cellular substrate for memory formation. Age-related declines in the expression of both NMDAR-dependent LTP and NMDAR subunit proteins in the CA1 region of the hippocampus have been well characterized and likely underlie agerelated memory impairment. In the current study, we examined NMDAR-dependent LTP in young Fischer 344 rats (4 months old) and aged rats (24 months old) given either a control diet or a diet supplemented with
\end{abstract}

S. J. Coultrap $(\bowtie) \cdot$ M. D. Browning

Department of Pharmacology, University of Colorado

Health Sciences Center,

MS 8303, P.O. Box 6511, Aurora, CO 80045, USA

e-mail: Steve.coultrap@uchsc.edu

P. C. Bickford

James A. Haley Veterans Administration Medical Center,

Tampa, FL, USA

P. C. Bickford

Center of Excellence for Aging and Brain Repair,

University of South Florida,

Tampa, FL, USA

P. C. Bickford

Department of Neurosurgery, University of South Florida,

Tampa, FL, USA

M. D. Browning

Program in Neuroscience,

University of Colorado Health Sciences Center,

Aurora, CO, USA blueberry extract for 6-8 weeks. NMDAR- dependent LTP was evoked by high-frequency stimulation (HFS) in the presence of nifedipine, to eliminate voltagegated calcium channel LTP. Field excitatory postsynaptic potentials (fEPSPs) were increased by $57 \% 1 \mathrm{~h}$ after HFS in young animals, but this potentiation was reduced to $31 \%$ in aged animals. Supplementation of the diet with blueberry extract elevated LTP (63\%) in aged animals to levels seen in young. The normalization of LTP may be due to the blueberry diet preventing a decline in synaptic strength, as measured by the slope of the fEPSP for a given fiber potential. The blueberry diet did not prevent age-related declines in NMDAR protein expression. However, phosphorylation of a key tyrosine residue on the NR2B subunit, important for increasing NMDAR function, was enhanced by the diet, suggesting that an increase in NMDAR function might overcome the loss in protein. This report provides evidence that dietary alterations later in life may prevent or postpone the cognitive declines associated with aging.

Keywords Anti-oxidant - Long-term potentiation · NMDA receptor $\cdot$ Phosphorylation $\cdot$ Synaptic strength

\section{Introduction}

Loss of cognitive function in the elderly presents an overwhelming challenge to the families of those afflicted. With America's demographic shift toward 
a more aged population, such mental declines will become an ever increasing challenge. In an effort to reduce the impact of age-related memory loss, intensive research efforts are underway to find ways to slow or reverse this cognitive impairment.

One aspect of cognitive function that appears to decline with age both in humans and in animal models is spatial learning (Barnes 1979, 1987; Rosenzweig and Barnes 2003). Several studies have focused on dietary alterations or supplementation to ameliorate the age-related loss of cognitive function in rat models (Stoll et al. 1993; Joseph et al. 1999; Eckles-Smith et al. 2000; Liu et al. 2002, 2003). In one such study, supplementation of the diet with extracts from blueberries, strawberries or spinach for eight weeks enhanced memory performance in the Morris water maze test (Joseph et al. 1999). While the beneficial behavioral effects of this diet in rats are clear, the cellular changes contributing to these effects have yet to be fully understood.

The major cellular mechanism thought to underlie spatial learning and memory is NMDAR-dependent long-term potentiation (LTP) in the hippocampus (Danysz et al. 1988; Morris 1989; Martin and Clark 2007). In the aged hippocampus voltage-gated calcium channel (VGCC)-dependent LTP is evoked by HFS, in addition to NMDAR-dependent LTP (Shankar et al. 1998). These two forms of LTP appear to oppose each other in learning and memory, blockade of NMDA receptors impairs (Danysz et al. 1988; Morris 1989), whereas blockade of VGCCs appears to enhance hippocampal dependent learning (Deyo et al. 1989; Meneses et al. 1997; Veng et al. 2003). In aged animals NMDAR-dependent LTP is reduced, while VGCCdependent LTP is enhanced (Shankar et al. 1998). Because of the increase in VGCC LTP, the deficits in NMDAR-dependent LTP are only apparent when VGCCs are blocked or weak induction paradigms are used (Shankar et al. 1998; Clayton et al. 2002; Moore et al. 1993; Deupree et al. 1993; Eckles-Smith et al. 2000).

The age-related impairment of NMDAR-dependent LTP is proposed to be due to decreases in NMDAR function (Barnes et al. 1997) and NMDAR subunit expression (Eckles-Smith et al. 2000; Clayton and Browning 2001; Sonntag et al. 2000; Kuehl-Kovarik et al. 2000). Consistent with this, we found that spermine, which enhances NMDAR function, rescued NMDA-dependent LTP in aged animals (Clayton et al. 2002). In another study from our lab, the age- related deficits in both NMDAR-dependent LTP and NR1 subunit expression were ameliorated by life-long caloric restriction (Eckles-Smith et al. 2000). Based on these previous studies, we chose to focus on agerelated changes in NMDAR-dependent LTP. Therefore, in the current study, we wanted to investigate whether a diet enriched with blueberry extract could overcome the biochemical and electrophysiological sequela associated with aging. Consistent with previous reports, we found deficits in both NMDARdependent LTP and synaptic strength in hippocampal slices from aged animals. In slices from animals given the blueberry-enriched diet, both NMDAR-dependent LTP and synaptic strength were elevated to levels seen in young controls. While the deficits in NMDAR subunit expression were not rescued by the diet, phosphorylation of a key tyrosine residue in the NMDAR was enhanced.

\section{Materials and methods}

Animals Five young adult (4 months old) and eight aged (22 months old) Fischer 344 rats were obtained from the National Institutes of Aging breading colony at Harlan Laboratories. Animals were housed two to three per cage in a climate-controlled environment with a 12-h light/dark cycle with water and rat chow available ad libitum. Aged animals were randomly divided into two groups of four animals: one received a control diet, while the other group received a diet enriched with blueberry extract for 6-8 weeks (Joseph et al. 1999). Aged animals were 23-24 months old at the time of experiment.

Tissue preparation Following euthanasia, the brain was removed to ice-cold oxygenated artificial cerebrospinal fluid (aCSF; $124 \mathrm{mM} \mathrm{NaCl}, 4 \mathrm{mM} \mathrm{KCl}$, $1 \mathrm{mM} \mathrm{MgCl} 2,2.5 \mathrm{mM} \mathrm{CaCl}_{2}, 10 \mathrm{mM}$ dextrose, $1 \mathrm{mM} \mathrm{KH}{ }_{2} \mathrm{PO}_{4}, 25.7 \mathrm{mM} \mathrm{NaHCO} 3$ ). Both hippocampi were removed, and the $\mathrm{CA} 1$ region was isolated as previously described (Clayton et al. 2002). Transverse CA1 mini-slices were made by chopping the CA1 $(400 \mu \mathrm{m})$ on a McIlwain tissue chopper. Slices were recovered for $90 \mathrm{~min}$ at $32^{\circ} \mathrm{C}$ in a perfusion chamber with aCSF (oxygenated with $95 \% \mathrm{O}_{2} / 5 \% \mathrm{CO}_{2}$ ) flowing at $3 \mathrm{ml} / \mathrm{min}$.

After recovery some slices were prepared for Western blot by sonication in $1 \%$ sodium dodecyl sulfate 
(SDS), $1 \mathrm{mM}$ ethylenediamineteraacetic acid, $10 \mathrm{mM}$ Tris (pH 8). Samples were heated to $95^{\circ} \mathrm{C}$ for $5 \mathrm{~min}$, and then frozen at $-80^{\circ} \mathrm{C}$. The remaining slices were kept in the perfusion chamber for electrophysiology.

Extracellular field recordings One or two slices from each animal were used for electrophysiological studies. Field excitatory post-synaptic potentials (fEPSPs) were obtained by stimulating the Schaffer collateral pathway using a bipolar tungsten stimulating electrode. The recording electrode, a silver chloride recording electrode inside a finely drawn glass capillary containing aCSF, was placed in the dendritic layer of the CA1. Stimuli were given at a rate of $0.033 \mathrm{~Hz}$. Both the initial slope and the peak amplitude of the resulting fEPSP were measured.

An input/output curve was obtained for each slice by increasing the stimulus intensity and measuring the resulting fiber potential and slope of the fEPSP. To compare input/output curves from different groups the fiber potential was measured and plotted against the initial slope of the corresponding fEPSP. Before the LTP experiment began, the applied stimulus was adjusted to produce an fEPSP that was $30-50 \%$ of the maximum amplitude. Baseline responses before potentiation had average initial slopes of $-160 \pm 22$, $-128 \pm 11$, and $-147 \pm 12 \mathrm{mV} / \mu$ s in young, aged control and aged blueberry diet animals, respectively. Baseline responses in aged control animals were slightly (but not significantly) lower than the other groups. Once a stable baseline was recorded for at least $20 \mathrm{~min}, 25 \mu \mathrm{M}$ nifedipine, the voltage-gated calcium channel (VGCC) blocker, was perfused for $30 \mathrm{~min}$. As previously described (Shankar et al. 1998), aged animals exhibit robust potentiation via VGCCs and this obscures NMDA-dependent LTP in these animals. LTP was then induced by four 1-s trains of $100-\mathrm{Hz}$ stimulation separated by $30 \mathrm{~s}$. Following this high frequency stimulation (HFS), nifedipine perfusion was then terminated. Responses were continuously recorded at $0.033 \mathrm{~Hz}$ for at least $1 \mathrm{~h}$ after HFS. LTP was measured as the percent change in the initial slope of the fEPSP at $1 \mathrm{~h}$ post HFS by comparing the fEPSP responses at 55-65 min with those spanning the 10 min prior to HFS, using one-way ANOVA with Tukey's post-hoc.

Semi-quantitative Western blotting Briefly, protein concentrations were determined by BCA assay
(Pierce). Samples were diluted to equal protein concentrations in sample loading buffer $(2.3 \% \mathrm{SDS}$, $67.5 \mathrm{mM}$ Tris, $10 \%$ glycerol, $5 \% \beta$-mercaptoethanol, $0.017 \%$ bromophenol blue). To allow comparison of samples run on different gels, each gel contained a five-point standard curve with 1.5-12 $\mu \mathrm{g}$ of whole hippocampal homogenate in sample loading buffer. The standards used on all gels in the study were from the same batch. Samples and standards in loading buffer were boiled for $5 \mathrm{~min}$ then loaded onto $7.5 \%$ polyacrylamide gels. Gels were run at $200 \mathrm{~V}$ until the bromophenol blue exited the gel. Proteins were transferred to polyscreen PVDF (New England Nuclear) using the Genie transfer apparatus (Idea Scientific). Blots were blocked with 3\% BSA in Trisbuffered saline (140 mM NaCl, $20 \mathrm{mM}$ Tris, $\mathrm{pH}$ 7.6) with $0.1 \%$ Tween 20 (TTBS) for $1 \mathrm{~h}$, then probed with primary antibody overnight at $4{ }^{\circ} \mathrm{C}$. Antibodies to NR1 (BD Pharmingen), NR2B (Snell et al. 1996), pY1472 NR2B (Phosphosolutions) and GluR2/3 (Chemicon) were diluted 1:3,000 in $1 \%$ BSA. Blots were probed with HRP-conjugated goat anti-mouse or goat anti-rabbit (Bio-Rad) antibodies and detected using Super Signal west dura ECL reagent (Pierce). Digital images of the blots were captured using a Chemiimager 4400 (Alpha Innotech). Briefly, each blot contained samples from young, aged control and aged blueberry diet in duplicate as well as a standard curve of hippocampal homogenate. An integrated density value (IDV) for samples and standards was determined as the sum of the intensity of the pixels constituting each band. A standard curve was generated by plotting the IDV of each standard by the micrograms of total protein loaded in that lane. The IDV of each sample was then compared with the standard curve to determine the immunoreactivity of that sample. Only bands that had an IDV within the standard curve were used. The immunoreactivity was divided by the amount of sample protein (in micrograms) loaded in each lane and reported in the figures.

Statistics One-way ANOVAs with Tukey's post-hoc were performed with GraphPad Prizm (GraphPad). Because two slices were used from several animals in the LTP study, a nested ANOVA was preformed with rat as a random factor nested in condition. This test confirmed that the variance was due to condition and not a clustering effect of rat as a subgroup within the condition group. 


\section{Results}

NMDAR-dependent LTP in the CA1 of aged rats

We examined the effects of a blueberry-enriched diet on NMDAR-dependent LTP in area CA1 of the hippocampus in aged animals. Previously, we reported that the LTP of population spikes induced by primed burst stimulation was absent in aged animals and that the deficit was relieved by a life-long calorically restricted diet (Eckles-Smith et al. 2000). Furthermore, we have shown that addition of certain berry extracts to the diet can slow the loss of function in memory tasks in aged rats (Joseph et al. 1999). In the current study, the decline of LTP in aged animals was examined by supplementation of the diet with blueberry extract later in life to determine the effectiveness of the diet for reducing the age-related decline in synaptic plasticity.

Field excitatory post-synaptic potentials (fEPSPs) were recorded from CA1 mini-slices of young (4 months old) and aged (24 months old) Fisher 344 rats. NMDAR-dependent LTP was induced by application of four trains of high-frequency stimulation (HFS) in the presence of nifedipine. Because VGCCdependent LTP is reportedly elevated in aged animals, slices were treated with nifedipine before and during LTP induction to insure that only NMDAR-dependent LTP was induced (Shankar et al. 1998). Nifedipine had no effect on the slope or amplitude of the fEPSP.

The initial slope of the fEPSP was measured before and after induction of LTP (Fig. 1). The degree of LTP was reported as the percent increase in fEPSP slope at $1 \mathrm{~h}$ after LTP induction relative to the fEPSP slope just before induction. NMDAR-dependent LTP was reduced in aged animals $(30.8 \pm 4 \%$ potentiation; Fig. 1) compared with young (57.2 $\pm 7 \%)$. LTP in aged animals receiving the blueberry enriched diet for 68 weeks was comparable with that seen in young animals $(63.3 \pm 9.5 \%)$. Potentiation was equivalent in young and aged animals given the blueberry diet, and both were elevated relative to aged control animals as determined by one-way ANOVA with Tukey's posthoc $[\mathrm{F}(2,19)=5.59, p<0.05]$.

Synaptic strength in the CA1 region of aged rats

It has been reported previously that there is a reduction in the fEPSP size relative to the fiber potential in aged animals, and this synaptic weakening may contribute to cognitive impairments (Barnes et al. 1992, 1997; Hsu et al. 2002; Deupree et al. 1991; Potier et al. 2000). To that end, we examined the input/output curve (I/O curve) by comparing the relationship between the fiber potential and the initial slope of the corresponding fEPSP. Figure 2a shows amplitude of the fiber potential relative to the stimulus intensity. The fiber potential generated for a given stimulus was slightly elevated in young animals relative to aged $[\mathrm{F}(2,17)=37.2, p<0.001$; $0.134 \pm 0.007,0.073 \pm 0.004$, and $0.086 \pm 0.004$ in young, aged controls, and aged blueberry diet, respectively]. There was, however, no difference between slices from aged animals given the control diet and those given the blueberry diet. As previously reported, there was a rightward shift of the input/output function in aged animals compared with young as measured by the slope of the I/O lines $(2,702 \pm 163$ in young vs $1,223 \pm 73$ in aged; Fig. 2). This reduction in synaptic strength was ameliorated in the aged animals given the blueberry enriched diet $(2,988 \pm 156)$, suggesting that this diet may prevent or delay this important synaptic change. The slope of the I/O curve was comparable in young and age animals on the blueberry diet, and both were significantly elevated from aged controls $[\mathrm{F}(2,17)=39.5, p<0.001)$. The loss of synaptic strength is postulated to represent a loss of functional synapses in the aged animal (Barnes et al. 1997; Rosenzweig and Barnes 2003). Thus, the age-related loss of synaptic strength and the amelioration of this loss by the blueberry diet may provide a mechanism for the decline of LTP with age and the subsequent normalization by the blueberry diet.

Age-related changes in NMDAR expression and phosphorylation

In addition to loss of synaptic strength, age-related decreases in expression of the NR1 and NR2B subunits of the NMDA receptor may also contribute to the LTP deficits (Eckles-Smith et al. 2000; Clayton and Browning 2001; Gemma et al. 2004). Therefore, we examined the expression of NMDA receptor subunit proteins from the CA1 mini-slices of young, aged, and aged animals give the blueberry diet. The expression of both NR1 and NR2B subunits was reduced in the aged animals compared with the young adults by $25 \%$ [Fig. 3, NR1: $\mathrm{F}(2,9)=5.17, p<0.05$, NR2B: $\mathrm{F}(2,9)=7.7, p<0.05]$. However, the blueberry-enriched diet failed to rescue the deficits in NR1 or NR2B expression $(p>0.05)$. 
Fig. 1 a-c Age-related deficit in NMDAR-dependent LTP in the CA1 is rescued by blueberry-enriched diet. a Sample traces showing EPSPs before and after HFS from young, aged control diet, and aged blueberry diet. The thin line depicts control EPSPs and the thicker line represents responses $60 \mathrm{~min}$ after HFS. b Time course of NMDARdependent LTP as measured by the initial slope of the EPSP in young (open circles; $n=8)$, aged control diet (closed triangles; $n=7$ ) and aged blueberry diet (closed squares; $n=7$ ). Baseline responses were obtained for 15 min with drugs and for $30 \mathrm{~min}$ in the presence of nifedipine. Then LTP was induced by application of four trains of 100$\mathrm{Hz}$ stimulation for $1 \mathrm{~s}$ per train, each train was $30 \mathrm{~s}$ apart. Nifedipine perfusion was terminated after the last train. c Potentiation of the slope of the EPSP at $60 \mathrm{~min}$ after HFS. LTP in aged control diet is significantly lower that in young or aged blueberry diet as determined by one-way ANOVA with Tukey's post-hoc analysis $\left({ }^{*} p<0.05\right)$
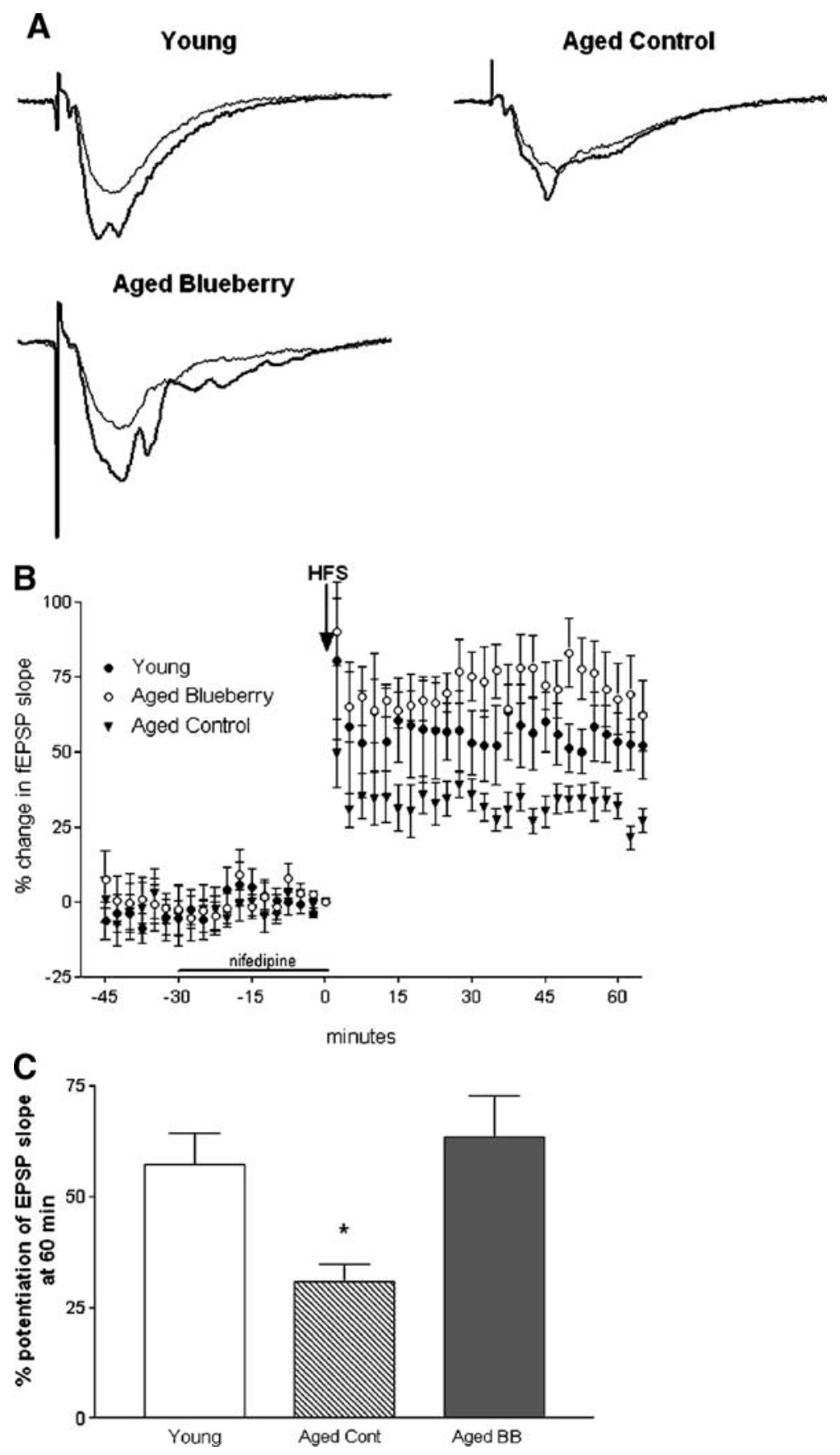

Expression of the AMPAR subunits GluR2/3 was not significantly altered by aging $[\mathrm{F}(2,9)=0.66, p>0.05]$.

NMDAR function is strongly regulated by phosphorylation (Wang and Salter 1994; Yu et al. 1997; Lu et al. 1999; Chen and Leonard 1996). Indeed, Norris et al. (1998a, b) suggested that reduced synaptic strength in aged animals could be due to a shift in the balance of protein kinase and phosphatase activities. Therefore, we investigated whether the blueberry diet could have modified NMDAR function via effects on phosphorylation. We examined the level of phosphorylation of NR2B on tyrosine 1472, a site thought to be important in regulation of receptor function (Nakazawa et al. 2001). Phosphorylation of 

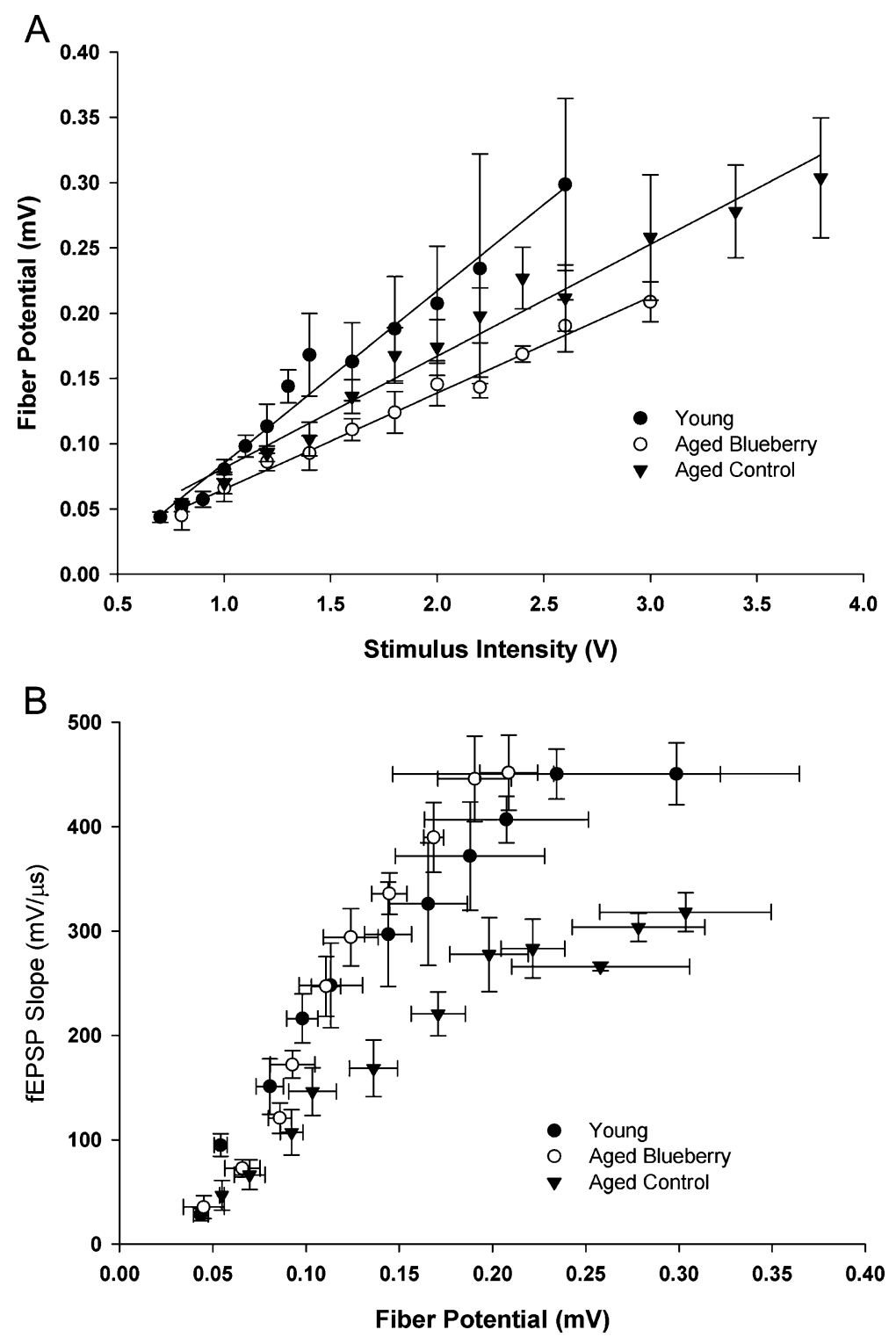

Fig. 2 a, b Age-related decrease in input/output curve is rescued by blueberry-enriched diet. a Stimulus intensity vs fiber potential in CA1 from young (closed circles; $n=8$ ), aged control diet (closed triangles; $n=6$ ) and aged blueberry diet animals (open circles; $n=6$ ). The fiber potential from a given stimulus intensity was reduced in aged animals on control or blueberry diet compared with young (the slope of the regression line was $0.134 \pm 0.007,0.073 \pm 0.004$, and $0.086 \pm 0.004$ in young, aged controls, and aged blueberry diet, respectively, $p<0.001$ young vs aged control and aged blueberry). b I/O curve showing initial slope of the EPSP as a function of the

this site was elevated, though not significantly, in aged animals compared with young. In aged animals given the blueberry enriched diet, however, there was a significant $[\mathrm{F}(2,9)=4.31, p<0.05] 90 \%$ increase in fiber potential evoked in young (closed circles; $n=8$ ), aged control diet (closed triangles; $n=6$ ) and aged blueberry diet (open circles; $n=6$ ). A linear regression was preformed on each group. The resulting slopes of the linear portion of the $\mathrm{I} / \mathrm{O}$ curves were $2,702 \pm 63,1,223 \pm 73$ and $2,988 \pm 156$ for young, aged control and aged blueberry, respectively. The resulting I/O curve slopes from the young and aged blueberry diet animals were significantly different from the aged control diet by oneway ANOVA with Tukey's post hoc $(p<0.001$ young vs aged control and aged blueberry vs aged control)

Y1472 phosphorylation compared with young animals (Fig. 4). In addition to the effects on synaptic strength, the blueberry diet could also contribute to the rescue of LTP in aged animals via an increase in 
A

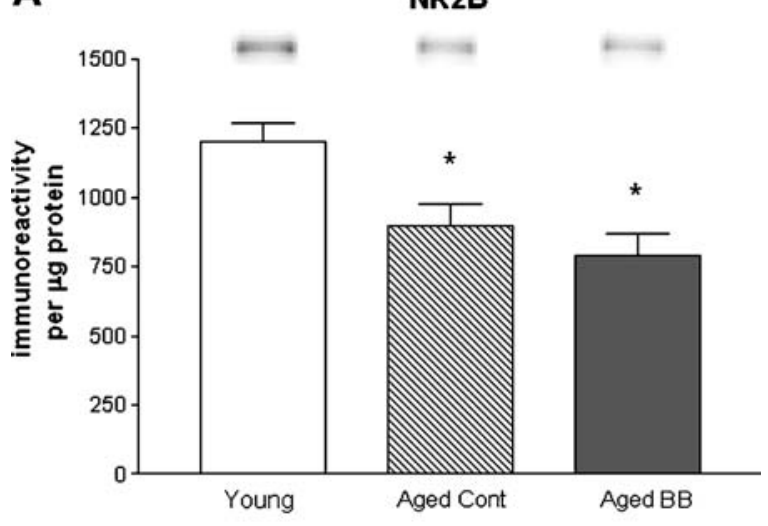

B

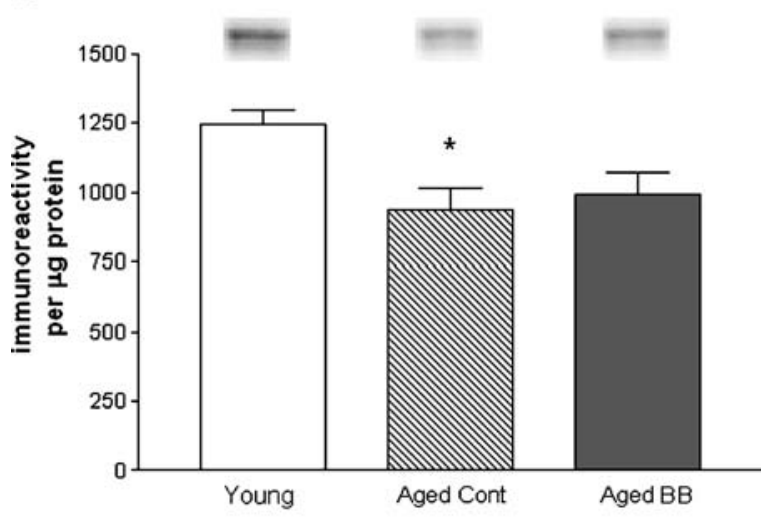

C

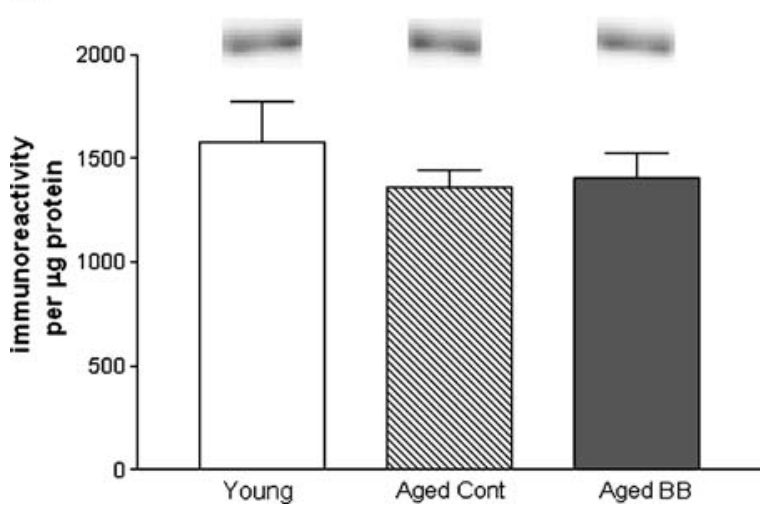

tyrosine phosphorylation of the NMDA receptor, which enhances function.

\section{Discussion}

In the current report, we show that LTP induced by HFS is reduced in aged animals when VGCCs are
Fig. 3 a-c Age-related decreases in NMDAR subunit expression is not rescued by blueberry-enriched diet as determined by Western blot. a NR2B subunit expression in young, aged control and aged blueberry was $1,199 \pm 67,895 \pm 82$ and $790 \pm$ 80 immunoreactivity units per $\mu \mathrm{g}$ protein, respectively $(n=4)$. Expression in aged animals was reduced by $25 \%$ compared with young (one-way ANOVA with Tukey's post-hoc, ${ }^{*} p<0.05$ young vs aged control and young vs aged blueberry). b NR1 subunit expression in young, aged control and aged blueberry was $1,243 \pm 52,936 \pm 78$ and $992 \pm 83$, respectively $(n=4)$. Expression in aged animals was reduced by $25 \%$ compared with young (one-way ANOVA with Tukey's post-hoc, ${ }^{*} p<0.05$ young vs AC). c Expression of GluR2/3 AMPAR subunits in young, aged control and aged blueberry was $1,577 \pm 196,1360 \pm 84$ and $1,405 \pm 120$, respectively $(n=4)$. Expression was not reduced in aged animals compared with young (one-way ANOVA $p>0.05$ )

blocked, providing further evidence that NMDARdependent LTP is reduced with aging. More importantly, the LTP deficit can be prevented by addition of blueberry extract to the diet for 6-8 weeks. This diet or other diets such as spinach or spirulina, which are also enriched in bioflavonoids, have previously been show to enhance the performance of aged rats in the Morris water maze (Joseph et al. 1999), enhance performance on a classical conditioning task (Cartford et al. 2002), reduce ischemic brain damage (Wang et al. 2005) and enhance the survival of transplanted neuronal tissue (Willis et al. 2005). It is well established that these diets can reduce markers of oxidative stress and inflammation (Gemma et al. 2002; Joseph et al. 1998). The reduction in oxidative

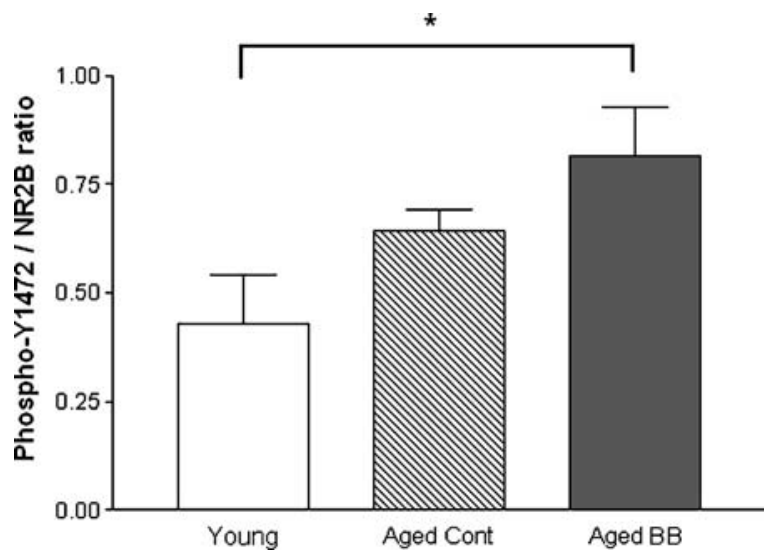

Fig. 4 Age-related increase in the phosphorylation of tyrosine 1472 on the NR2B subunit. The ratio of phospho-Tyr1472 immunoreactivity to NR2B immunoreactivity was $0.43 \pm 0.11$, $0.64 \pm 0.04$, and $0.82 \pm 0.11$ in young, aged control and aged blueberry, respectively ( $n=4$; one-way ANOVA with Tukey's post-hoc, $* p<0.05$ young vs aged blueberry) 
damage to proteins, membranes and DNA is likely to be the mechanisms by which these diets can lead to functional improvement in several domains. For example, in the cerebellum a functional improvement in the $\beta$-adrenergic receptor is linked with the improvement in classical conditioning, and this is also associated with decreased oxidative damage markers and pro-inflammatory (Cartford et al. 2002; Gemma et al. 2002). Thus, the mechanism underlying the improvement in LTP may be related to increased function of receptor signaling involved in NMDARdependent LTP.

NMDAR-dependent LTP in the CA1 region of the hippocampus is thought to be the cellular mechanism responsible for spatial memory formation (Barnes 1987). In aged animals, two types of LTP are induced by high-frequency stimulation: NMDAR-dependent LTP and VGCC-dependent LTP (Shankar et al. 1998). NMDAR-dependent LTP is thought to contribute to memory formation, while VGCC-dependent LTP is thought to be detrimental to memory formation (Shankar et al. 1998; Norris et al. 1998b). There is an age-related decline in NMDAR-dependent LTP coincident with an increase in VGCC-dependent LTP (Shankar et al. 1998). Consistent with these changes in the mechanisms of LTP induction, age-related alterations in the expression of NMDARs and VGCCs in the CA1 have been identified. Expression of the NR1 and NR2B subunits of the NMDA receptor decline with age leading to a reduction in NMDAR function, while expression of the VGCC $\alpha_{1 \mathrm{D}}$ subunits increase with age in area CA1 (Eckles-Smith et al. 2000; Clayton and Browning 2001; Clayton et al. 2002; Barnes et al. 1997; Potier et al. 2000; Veng et al. 2003; Veng and Browning 2002). In fact, antisense knockdown of NR2B expression in young animals reduced LTP in a manner similar to aging (Clayton et al. 2002). We have previously reported that lifelong caloric restriction prevented the age-related deficits in NMDAR-dependent LTP expression as well as the reduction in NMDAR expression (Eckles-Smith et al. 2000). In the current study, administration of a blueberry enriched diet later in life prevented the deficit in NMDAR-dependent LTP, but did not prevent the age-related loss of NMDA receptors. One possibility is that the function of the existing NMDARs is somehow enhanced following the blueberry diet. Consistent with this, we report an increase in the phosphorylation of tyrosine 1472 on the NR2B subunit. Tyrosine phosphorylation of NMDARs has previously been reported to increase receptor function (Wang and Salter 1994; Chen and Leonard 1996; Yu et al. 1997; Lu et al. 1999). Phosphorylation of Y1472 in particular has been implicated in LTP (Nakazawa et al. 2001). Thus, the increase in tyrosine phosphorylation of NR2B associated with the blueberry diet may result in an increase in NMDAR function. Such an increase in function could provide a mechanism to compensate for the loss of NMDA receptors.

In addition to the age-related decline in LTP, we and others also found age-related deficiencies in the I/O curve of the fEPSP (Barnes et al. 1992, 1997; Hsu et al. 2002; Deupree et al. 1991; Potier et al. 2000). The changes underlying the loss of synaptic strength are a matter of some debate. Similar changes in the dentate gyrus are likely due to a decrease in the number of synapses; however, in the CA1 there are no significant age-related losses of principal cells nor are there reductions in the number of synapses (Rapp and Gallagher 1996; Rasmussen et al. 1996; Geinisman et al. 1995, 2004). It has been proposed that the reduction in synaptic strength is due to a decrease in functional synapses in older rats (Barnes et al. 1992; Geinisman 1999). This decrease is not likely due to presynaptic changes as synaptic proteins involved in neurotransmitter release, such as synaptophysin, synaptotagmin, SNAP-25 and synapsin, are not altered (Nicolle et al. 1999). Likewise, studies from several laboratories have concluded that there is no change in paired pulse facilitation ratios with age in the CA1 (Deupree et al. 1993; Landfield and Lynch 1977; Landfield et al. 1978). Thus, the mechanism for the age-related decrease in the fEPSP is likely postsynaptic. We report here that expression of the AMPAR subunits GluR1 and 2 is not altered in aged animals, confirming findings from previous studies (Eckles-Smith et al. 2000; Clayton and Browning 2001; Nicolle et al. 1999). Sensitivity of synaptic responses to AMPA is reportedly reduced in aged animals (Barnes et al. 1992). One study found that the age-related reduction in synaptic strength was due to a shift in the balance of protein kinase and phosphatase activities (Norris et al. 1998a). In this study, blockade of serine/threonine phosphatase activity increased synaptic strength in aged animals. The function of AMPA receptors is thought to be enhanced by phosphorylation (Boehm and Malinow 2005). It is 
possible that the functional expression of AMPARs in the synaptic membrane or the conductance of existing AMPARs is reduced with aging, resulting in a weakened AMPA current (Geinisman 1999). Further studies will be needed to determine if this is the case. In the current study, we found that the age-related decrease in synaptic strength is prevented by the blueberry enriched diet, although the mechanism for this decline remains to be elucidated.

While lifelong caloric restriction may prevent or delay the onset of the decline in LTP by preventing the loss of NMDARs, supplementation of the diet with blueberry extract later in life may provide a means to offset the decline in NMDAR expression by enhancing cellular mechanisms which compensate for the loss. This diet has previously been shown to reverse age-related declines in cognitive and motor behavior (Joseph et al. 1999). Here we show that agerelated deficits in NMDAR-dependent LTP, a cellular substrate for learning and memory, are also prevented by the blueberry enriched diet. The normalization of LTP was not due to reversal of NMDAR subunit loss, but may in fact be due to preventing the loss of synaptic strength or by producing an increase in NMDAR function resulting from increases in tyrosine phosphorylation of the NR2B subunit.

Acknowledgements We would like to thank Dr. Ronald Freund and Tianna Hicklin for helpful discussions on this work. This work was supported by National Institutes of Health Grants: AG04418, AA09675, AA014619 and the VA Medical Research Service. M.D.B. has a financial interest in Phosphosolutions, which provided some of the antibodies used in this study.

Open Access This article is distributed under the terms of the Creative Commons Attribution Noncommercial License which permits any noncommercial use, distribution, and reproduction in any medium, provided the original author(s) and source are credited.

\section{References}

Barnes CA (1979) Memory deficits associated with senescence: a neurophysiological and behavioral study in the rat. $\mathrm{J}$ Comp Physiol Psychol 93:74-104

Barnes CA (1987) Neurological and behavioral investigations of memory failure in aging animals. Int $\mathrm{J}$ Neurol 2122:130-136

Barnes CA, Rao G, Foster TC, McNaughton BL (1992) Region-specific age effects on AMPA sensitivity: electrophysiological evidence for loss of synaptic contacts in hippocampal field CA1. Hippocampus 2:457-468
Barnes CA, Rao G, Shen J (1997) Age-related decrease in the $\mathrm{N}$-methyl-aspartateR-mediated excitatory postsynaptic potential in hippocampal region CA1. Neurobiol Aging 18:445-452

Boehm J, Malinow R (2005) AMPA receptor phosphorylation during synaptic plasticity. Biochem Soc Trans 33:13541356

Cartford MC, Gemma C, Bickford PC (2002) Eighteen-monthold Fischer 344 rats fed a spinach-enriched diet show improved delay classical eyeblink conditioning and reduced expression of tumor necrosis factor alpha (TNFalpha) and TNFbeta in the cerebellum. J Neurosci 22:58135816

Chen C, Leonard JP (1996) Protein tyrosine kinase-mediated potentiation of currents from cloned NMDA receptors. J Neurochem 67:194-200

Clayton DA, Browning MD (2001) Deficits in the expression of the NR2B subunit in the hippocampus of aged Fisher 344 rats. Neurobiol Aging 22:165-168

Clayton DA, Mesches MH, Alvarez E, Bickford PC, Browning MD (2002) A hippocampal NR2B deficit can mimic agerelated changes in long-term potentiation and spatial learning in the Fischer 344 Rat. J Neurosci 22:3628-3637

Danysz W, Wroblewski JT, Costa E (1988) Learning impairment in rats by N-methyl-D-aspartate receptor antagonists. Neuropharmacology 27:653-656

Deupree DL, Turner DA, Watters CL (1991) Spatial performance correlates with in vitro potentiation in young and aged Fischer 344 rats. Brain Res 554:1-9

Deupree DL, Bradley J, Turner DA (1993) Age-related alterations in potentiation in the CA1 region in F344 rats. Neurobiol Aging 14:249-258

Deyo RA, Straube KT, Disterhoft JF (1989) Nimodipine facilitates associative learning in aging rabbits. Science 243:809-811

Eckles-Smith K, Clayton D, Bickford P, Browning MD (2000) Caloric restriction prevents age-related deficits in LTP and in NMDA receptor expression. Brain Res Mol Brain Res 78:154-162

Geinisman Y (1999) Age-related decline in memory function: is it associated with a loss of synapses? Neurobiol Aging 20:353-356

Geinisman Y, Detoledo-Morrell L, Morrell F, Heller RE (1995) Hippocampal markers of age-related memory dysfunction: behavioral, electrophysiological and morphological perspectives. Prog Neurobiol 45:223-252

Geinisman Y, Ganeshina O, Yoshida R, Berry RW, Disterhoft JF, Gallagher M (2004) Aging, spatial learning, and total synapse number in the rat CA1 stratum radiatum. Neurobiol Aging 25:407-416

Gemma C, Mesches MH, Sepesi B, Choo K, Holmes DB, Bickford PC (2002) Diets enriched in foods with high antioxidant activity reverse age-induced decreases in cerebellar beta-adrenergic function and increases in proinflammatory cytokines. J Neurosci 22:6114-6120

Gemma C, Stellwagen H, Fister M, Coultrap SJ, Mesches MH, Browning MD et al (2004) Rosiglitazone improves contextual fear conditioning in aged rats. Neuroreport $15: 2255-2259$

Hsu KS, Huang CC, Liang YC, Wu HM, Chen YL, Lo SW et al (2002) Alterations in the balance of protein kinase and 
phosphatase activities and age-related impairments of synaptic transmission and long-term potentiation. Hippocampus 12:787-802

Joseph JA, Denisova N, Fisher D, Bickford P, Prior R, Cao G (1998) Age-related neurodegeneration and oxidative stress: putative nutritional intervention. Neurol Clin 16:747-755

Joseph JA, Shukitt-Hale B, Denisova NA, Bielinski D, Martin A, McEwen JJ et al (1999) Reversals of age-related declines in neuronal signal transduction, cognitive, and motor behavioral deficits with blueberry, spinach, or strawberry dietary supplementation. J Neurosci 19:81148121

Kuehl-Kovarik MC, Magnusson KR, Premkumar LS, Partin KM (2000) Electrophysiological analysis of NMDA receptor subunit changes in the aging mouse cortex. Mech Ageing Dev 115:39-59

Landfield PW, Lynch G (1977) Impaired monosynaptic potentiation in in vitro hippocampal slices from aged, memory-deficient rats. J Gerontol 32: 523-533

Landfield PW, McGaugh JL, Lynch G (1978) Impaired synaptic potentiation processes in the hippocampus of aged, memory-deficient rats. Brain Res 150: 85-101

Liu J, Atamna H, Kuratsune H, Ames BN (2002) Delaying brain mitochondrial decay and aging with mitochondrial antioxidants and metabolites. Ann N Y Acad Sci 959:133166

Liu R, Liu IY, Bi X, Thompson RF, Doctrow SR, Malfroy B et al (2003) Reversal of age-related learning deficits and brain oxidative stress in mice with superoxide dismutase/ catalase mimetics. Proc Natl Acad Sci USA 100:85268531

Lu WY, Xiong ZG, Lei S, Orser BA, Dudek E, Browning MD et al (1999) G-protein-coupled receptors act via protein kinase $\mathrm{C}$ and $\mathrm{Src}$ to regulate NMDA receptors. Nat Neurosci 2:331-338

Martin SJ, Clark RE (2007) The rodent hippocampus and spatial memory: from synapses to systems. Cell Mol Life Sci 64:401-431

Meneses A, Terron J, Ibarra M, Hong E (1997) Effects of nimodipine on learning in normotensive and spontaneously hypertensive rats. Behav Brain Res 85:121-125

Moore CI, Browning MD, Rose GM (1993) Hippocampal plasticity induced by primed burst, but not long-term potentiation, stimulation is impaired in area CA1 of aged Fischer 344 rats. Hippocampus 3:57-66

Morris RG (1989) Synaptic plasticity and learning: selective impairment of learning rats and blockade of long-term potentiation in vivo by the N-methyl-D- aspartate receptor antagonist AP5. J Neurosci 9:3040-3057

Nakazawa T, Komai S, Tezuka T, Hisatsune C, Umemori H, Semba K et al (2001) Characterization of Fyn-mediated tyrosine phosphorylation sites on GluR epsilon 2 (NR2B) subunit of the N-methyl-D-aspartate receptor. J Biol Chem 276:693-699

Nicolle MM, Gallagher M, McKinney M (1999) No loss of synaptic proteins in the hippocampus of aged, behaviorally impaired rats. Neurobiol Aging 20:343-348
Norris CM, Halpain S, Foster TC (1998a) Alterations in the balance of protein kinase/phosphatase activities parallel reduced synaptic strength during aging. J Neurophysiol 80:1567-1570

Norris CM, Halpain S, Foster TC (1998b) Reversal of agerelated alterations in synaptic plasticity by blockade of Ltype Ca2+ channels. J Neurosci 18:3171-3179

Potier B, Poindessous-Jazat F, Dutar P, Billard JM (2000) NMDA receptor activation in the aged rat hippocampus. Exp Gerontol 35:1185-1199

Rapp PR, Gallagher M (1996) Preserved neuron number in the hippocampus of aged rats with spatial learning deficits. Proc Natl Acad Sci USA 93:9926-9930

Rasmussen T, Schliemann T, Sorensen JC, Zimmer J, West MJ (1996) Memory impaired aged rats: no loss of principal hippocampal and subicular neurons. Neurobiol Aging 17:143-147

Rosenzweig ES, Barnes CA (2003) Impact of aging on hippocampal function: plasticity, network dynamics, and cognition. Prog Neurobiol 69:143-179

Shankar S, Teyler TJ, Robbins N (1998) Aging differentially alters forms of long-term potentiation in rat hippocampal area CA1. J Neurophysiol 79:334-341

Snell LD, Nunley KR, Lickteig RL, Browning MD, Tabakoff B, Hoffman PL (1996) Regional and subunit specific changes in NMDA receptor mRNA and immunoreactivity in mouse brain following chronic ethanol ingestion. Brain Res Mol Brain Res 40: 71-78

Sonntag WE, Bennett SA, Khan AS, Thornton PL, Xu X, Ingram RL et al (2000) Age and insulin-like growth factor-1 modulate N-methyl-D-aspartate receptor subtype expression in rats. Brain Res Bull 51:331-338

Stoll S, Hartmann H, Cohen SA, Mnller WE (1993) The potent free radical scavenger alpha-lipoic acid improves memory in aged mice: putative relationship to NMDA receptor deficits. Pharmacol Biochem Behav 46:799-805

Veng LM, Browning MD (2002) Regionally selective alterations in expression of the alpha(1D) subunit $(\mathrm{Ca}(\mathrm{v}) 1.3)$ of L-type calcium channels in the hippocampus of aged rats. Brain Res Mol Brain Res 107:120-127

Veng LM, Mesches MH, Browning MD (2003) Age-related working memory impairment is correlated with increases in the L-type calcium channel protein alpha1D (Cav1.3) in area CA1 of the hippocampus and both are ameliorated by chronic nimodipine treatment. Brain Res Mol Brain Res 110:193-202

Wang YT, Salter MW (1994) Regulation of NMDA receptors by tyrosine kinases and phosphatases. Nature 369:233-235

Wang Y, Chang CF, Chou J, Chen HL, Deng X, Harvey BK et al (2005) Dietary supplementation with blueberries, spinach, or spirulina reduces ischemic brain damage. Exp Neurol 193:75-84

Willis L, Bickford P, Zaman V, Moore A, Granholm AC (2005) Blueberry extract enhances survival of intraocular hippocampal transplants. Cell Transplant 14:213-223

Yu XM, Askalan R, Keil GJ, Salter MW (1997) NMDA channel regulation by channel-associated protein tyrosine kinase Src. Science 275:674-678 